\title{
Análise dos Aspectos Epidemiológicos da Malária na região Nordeste do Brasil
}

\section{Analysis of the Epidemiological Aspects of Malaria in Northeastern Brazil}

Caio Willer Brito Gonçalves ${ }^{1}$, Renan Alves Rodrigues ${ }^{2}$, Adir Bernardes Pinto Neto $^{3}$, Dario Luigi Ferraz Gomes ${ }^{4}$, Mailane da Silva ${ }^{5}$, Gabriel Viana Boa sorte ${ }^{6}$

\section{RESUMO}

Os estudos epidemiológicos da ocorrência da malária são de suma importância para a detecção de surtos e epidemia, visando contribuir para o maior conhecimento e controle oportuno da doença em diferentes regiões do Brasil, visto que cada região possui sua fauna específica de Anopheles. Esse estudo teve por objetivo analisar os aspectos epidemiológicos da malária no nordeste do Brasil. Estudo transversal, retrospectivo com abordagem quantitativa e descritiva por meio da consulta à base de dados do Sistema de Informação de Agravo de Notificação do Ministério da Saúde no período de janeiro de 2015 a dezembro de 2019. Foram verificadas 490 notificações de malária, sendo 377 casos do sexo feminino. Foi identificado um maior número de casos na idade entre 20 a 29 anos, totalizando 250 casos diagnosticados. Já em relação a cor foram 323 casos da etnia parda. Os resultados deste estudo possibilitaram descrever o perfil epidemiológico da malária nos estados do nordeste brasileiro, demostrando que apesar dos esforços do MS ainda encontra-se casos notificados sem o total controle da cadeia de transmissão da doença.

Palavras-chave: Monitoramento Epidemiológico. Malária. Transmissão.

\section{ABSTRACT}

Epidemiological studies on the occurrence of malaria are of paramount importance for detecting outbreaks and epidemics, aiming to contribute to greater knowledge and timely control of the disease in different regions of Brazil, since each region has its specific Anopheles fauna. This study aimed to analyze epidemiological aspects of malaria in northeastern Brazil. Cross-sectional, retrospective study with a quantitative and descriptive approach by consulting the database of the Ministry of Health Notification Information System from January 2015 to February 2019. 490 malaria notifications were verified, 377 of which were cases female. A greater number of cases was identified between 20 and 29 years old, totaling 250 diagnosed cases. Regarding color, there were 323 cases of mixed race. The results of this study made it possible to describe the epidemiological profile of malaria in the states of northeastern Brazil, showing that despite the efforts of the Ministry of Health, there are still cases reported without full control of the disease transmission chain.

Keywords: Epidemiological Monitoring. Malaria. Transmission.
${ }^{1}$ Discente do curso de medicina da Universidade de Gurupi.

E-mail: caiowillerb@gmail.com

2 Docente do curso de medicina da Universidade de Gurupi.

${ }^{3}$ Discente do curso de medicina da Universidade de Gurupi.

${ }^{4}$ Discente do curso de medicina da Universidade de Gurupi.

${ }^{5}$ Discente do curso de medicina da Universidade de Gurupi.

${ }^{6}$ Discente do curso de medicina da Universidade de Gurupi. 


\section{INTRODUÇAOO}

Também conhecida como maleita, impaludismo, paludismo e febre terçã ou quartã, a malária representa, desde a Antiguidade, um dos principais flagelos da humanidade, em que pelo menos 300 milhões de pessoas contraem malária por ano em todo o mundo. Destas, cerca de 1 a 2 milhões morrem. A malária está presente, também, em mais de 90 países, sendo os mais prevalentes a Índia e o Brasil com cerca de 300 mil casos/ano, seguidos do Afeganistão e China. ${ }^{1}$

A ocorrência de malária está intimamente associada à presença e proliferação de mosquitos do gênero Anopheles, onde cada região possui sua fauna específica em que influencia diretamente na incidência dos casos. No Brasil, os mosquitos transmissores da malária nas regiões costeiras, e particularmente na Mata Atlântica, eram o $A$. cruzi, o $A$. bellator e $\circ A$. aquasalis. Atualmente estes mosquitos têm importância epidemiológica apenas potencial. Mas, por todo o interior do país, incluídas as capitais, a principal espécie transmissora sempre foi o Anopheles darlingi, que hoje, ausente das áreas urbanizadas brasileiras, está restrito à Amazônia. ${ }^{2}$

Os estudos epidemiológicos da ocorrência da malária são essenciais para a detecção de surtos e epidemias, visando contribuir para o maior conhecimento das cadeias de transmissão da doença em diferentes regiões do Brasil.

Neste sentido, esta pesquisa teve por objetivo analisar os aspectos epidemiológicos da malária notificada na região do Nordeste do Brasil, na amostra que abrange de janeiro de 2015 a dezembro de 2019, no intuito de fornecer informações ao poder público para um melhor planejamento de ações visando a redução da cadeia de transmissão.

\section{MATERIAIS E METODOS}

Estudo epidemiológico, transversal, retrospectivo, descritivo com apresentação quantitativa, consultado com informações disponíveis no Sistema de Informação de Agravos de Notificação (SINAN).

Os critérios para inclusão no estudo foram os casos diagnosticados com malária em estados do Nordeste, entre janeiro de 2015 a dezembro de 2019, registradas no SINAN. Dessa forma, não houve a necessidade de aprovação no Comitê de Ética em Pesquisa 
em Humanos, segundo a Lei $N^{\circ}$ 466/2012, por se tratar de dados já previamente coletados.

Foram excluídas do estudo as notificações inexistentes como as do estado do Maranhão que não foram registradas no sistema, as notificações incompletas com registro fora da amostra pesquisada e as variáveis não analisadas no estudo. Abordou-se como fonte de informações o SINAN, que apresenta dados das notificações e investigações dos casos de patologias e suas complicações, que constam da lista nacional de notificação.

As variáveis analisadas foram números de casos notificados, sexo, faixa etária, raça, meses mais afetados e relacionados à estação, estados mais prevalentes, números de casos por capital nordestina e números de casos a cada 100mil habitantes. Estas variáveis, de acordo com a experiência dos pesquisadores do estudo são as questões mais adequadas para compreender as características dos infectados pela doença no estado. Os dados foram coletados por dois pesquisadores independentes a partir de planilhas criadas pelo aplicativo TabWin32 na versão 3.6b e encaminhados para os programas Microsoft Excel 2013 que permitiu a análise.

\section{RESULTADOS}

Ao todo foram notificados 489 casos de malária entre 2015 a 2019 na região Nordeste do Brasil (Figura 1). Observa-se um aumento significativo do número de casos notificados nos 2018, concentrando $31,2 \%$ dos casos, porém, poderia haver um maior número de ocorrências se os dados do estado do Maranhão tivessem disponíveis na plataforma de pesquisa do SINAN.

Figura 1. Notificações de casos de malária distribuídas por ano da região Nordeste de 2015 a 2019.

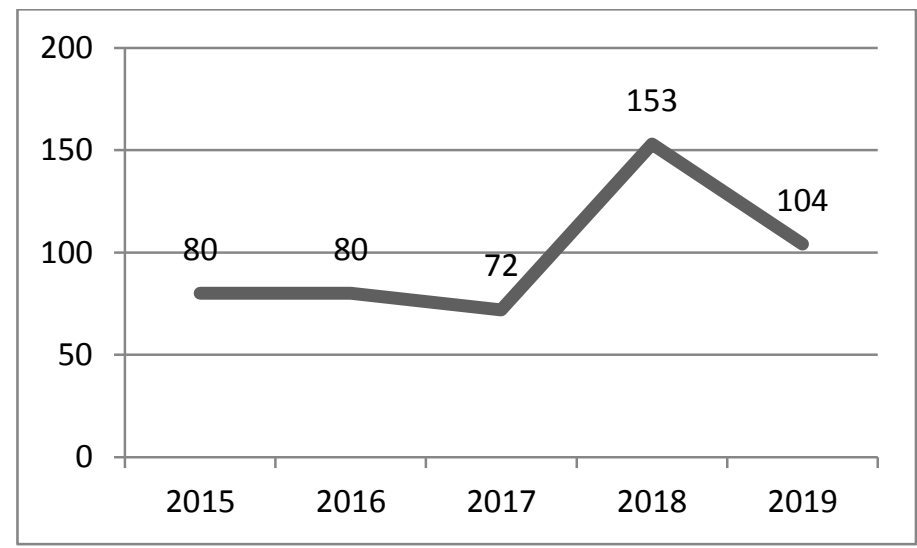

Fonte: (SINAN, 2020) 
DOI: 10.18606/2318-1419/amazonia.sci.health.v8n2p42-50
GONÇALVES, CWB. RODRIGUES, RA. NETO, ABP. GOMES, DLF, SILVA' M. SORTE, GVB.

Análise dos Aspectos Epidemiológicos da Malária na região Nordeste do Brasil

Em relação a faixa etária (Tabela 1), os notificados entre 20 e 39 anos foram os mais acometidos, aproximadamente $50 \%$, seguido da população entre 40 e 59 anos com $31,5 \%$.

Tabela 1. Distribuição de casos de Malária por faixa etária na região Nordeste dentre o período de 2015 até 2019.

\begin{tabular}{cc}
\hline Faixa etária & $\mathbf{N}^{\circ}$ de casos \\
\hline$<1$ ano & 4 \\
$1--4$ & 10 \\
$5--9$ & 6 \\
$10--14$ & 9 \\
$15--19$ & 26 \\
$20--39$ & 250 \\
$40--59$ & 153 \\
$60--64$ & 13 \\
$65--69$ & 7 \\
$70--79$ & 8 \\
$80+$ & 3 \\
\hline
\end{tabular}

Fonte: (SINAN, 2020)

Em relação aos resultados parasitológicos dos casos acometidos pela malária, observa-se que o Plasmidium vivax obteve 353 casos, e os menos acometidos foram FG (gametócitos de $P$. falciparum) e Malariae com apenas um caso cada. Dados apresentados na tabela 2 .

Tabela 2. Relação entre os números de casos de Malária e o resultado parasitológico na região Nordeste, no período de 2015 até 2020.

\begin{tabular}{cc}
\hline Resultado parasitológico & Quantidade \\
\hline Falciparum & 104 \\
F + FG & 3 \\
Vivax & 353 \\
F + V & 19 \\
V + FG & 4 \\
FG & 1 \\
Malariae & 1 \\
\hline
\end{tabular}

Fonte: (SINAN, 2020) 
DOI: 10.18606/2318-1419/amazonia.sci.health.v8n2p42-50
GONÇALVES, CWB. RODRIGUES, RA. NETO, ABP. GOMES, DLF, SILVA' M. SORTE, GVB.

Análise dos Aspectos Epidemiológicos da Malária na região Nordeste do Brasil

Os estados mais prevalentes (Figura 2) foram a Bahia com 145 casos, seguido do Piauí com 95 casos. Já os menos prevalentes foram os estados de Alagoas e Sergipe com respectivamente 15 e 16 casos.

Figura 2 - Distribuição dos casos de Malária por unidade federativa do Nordeste no período de 2015 até 2019.

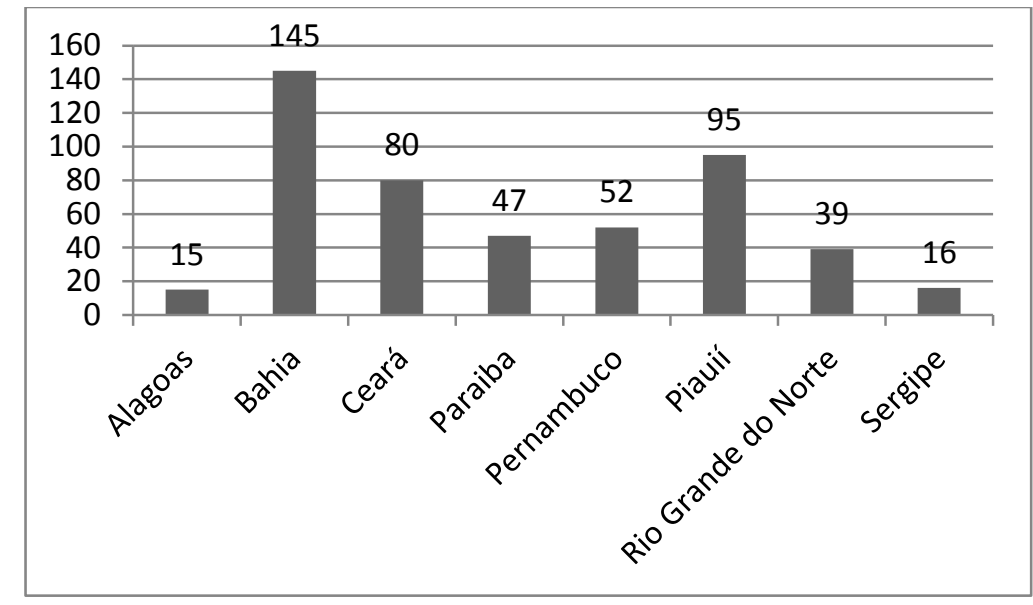

Fonte: (SINAN, 2020)

$\mathrm{Na}$ relação entre números de habitantes com os números notificados em cada estado, observou-se que o estado do Piauí foi estado mais acometido com aproximadamente 3 casos a cada 100 mil habitantes e em relação ao estado menos acometido, observou-se o Alagoas com 0,45 caso a cada 100 mil habitantes. Dados apresentados na figura 3 .

Figura 3 - Número de casos de Malária a cada 100 mil habitantes por unidade federativa no Nordeste no período de 2015 até 2020.

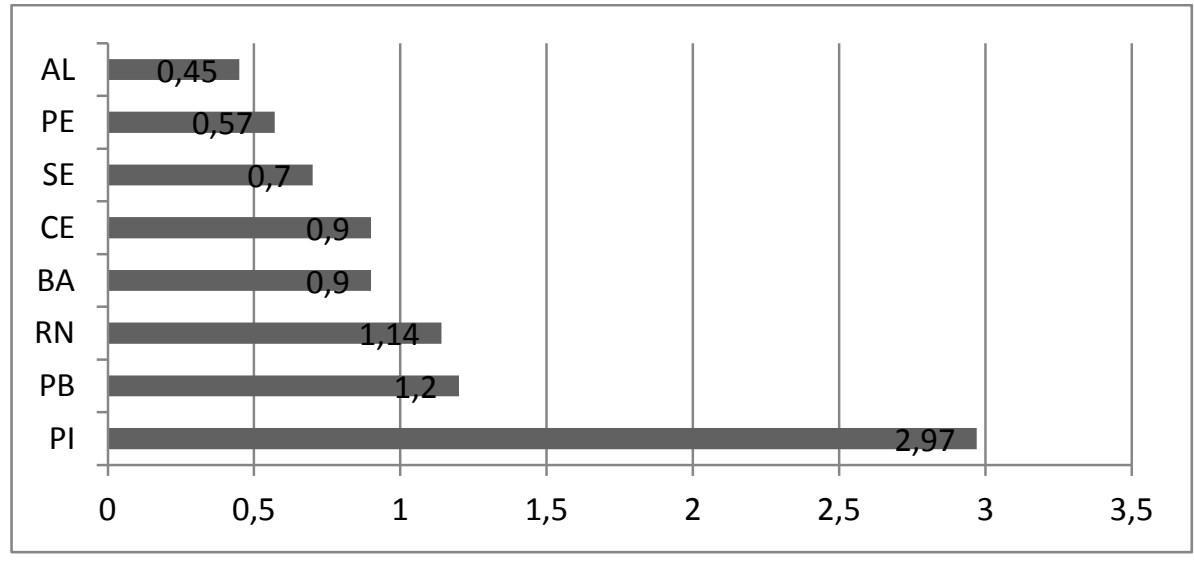

Fonte: (SINAN, 2020) 


\section{DISCUSSAOO}

A malária representa um grave problema de saúde pública no mundo e está presente em 91 países. A Região Africana da OMS foi responsável pela maioria dos casos globais de malária (90\%), seguida pela Região do Sudeste Asiático $(7 \%)$ e pela Região do Mediterrâneo Oriental (2\%). ${ }^{3}$

No Brasil, a grande totalidade dos casos ocorre na Amazônia brasileira, sendo uma doença causada por protozoários do gênero Plasmodium e transmitida pelos insetos do gênero Anopheles. ${ }^{4,5}$

A transmissão fora desta região, por sua vez, é rara e está restrita a pequenos focos residuais ou novos focos com baixas taxas de transmissão, assim como, é demonstrado em nosso estudo, a partir dos números de casos registrados na região nordeste, os quais são inferiores ao número de casos notificados na Amazônia. As existências desses focos são o resultado da reintrodução do parasita a partir do fluxo de pessoas advindas de áreas endêmicas de outras regiões do país, e à manutenção do vetor transmissor em algumas regiões, onde os riscos para ocorrência de casos autóctones da doença necessitam de atenção sanitária adequada, evitando assim, a ampliação dos focos existentes. ${ }^{6,7}$

No nordeste brasileiro, o maior número de casos foi registrado para as faixas etárias de 20 a 39 e 40 a 59 anos, as quais, assim como demonstrado em outros estudos, concentram uma grande quantidade de indivíduos economicamente ativos, sugerindo que as atividades ocupacionais dos mesmos, principalmente aquelas relacionadas à agricultura, garimpo e extrativismo vegetal possam influenciar na transmissão da malária, tornando estes indivíduos mais suscetíveis à contaminação. Verifica-se, uma menor frequência de casos em maiores de 60 anos, grupo com potencial susceptibilidade, visto que muitos possuem saúde precária devido à diminuição da imunidade inerente à própria idade. ${ }^{5,8-10}$

No período estudado, há um predomínio de malária causada por $P$. vivax, não sendo diferente do que se observa nos demais estados da Amazônia brasileira, inclusive, esta espécie, também é a mais prevalente em outras regiões do Brasil. 5, 8-10

Entre os estados mais acometidos nesta região, o estado da Bahia apresentou o maior número de casos registrados. Segundo o boletim epidemiológico da situação da malária no estado da Bahia de 2018 a 2019, sua carta anofélica demonstra a existência 
de uma ampla dispersão de espécies de anofelinos com importância epidemiológica, o que denota alta receptividade à transmissão vetorial do Plasmodium $s p .{ }^{8}$

Além da dispersão de insetos do gênero Anopheles, ressalta-se a vulnerabilidade de diferentes municípios à introdução ou reintrodução da malária, determinada por desequilíbrios ambientais e/ou sociais relacionados à mineração, extrativismo vegetal ou situações análogas. Ainda assim, boa parte dos casos registrados se devem ao fluxo constante de indivíduos doentes ou infectados, procedentes de áreas endêmicas, como a região Amazônica ou, inclusive, de outros países, principalmente do continente Africano. ${ }^{8}$

Apesar de que neste estudo não foram incluídos dados referentes ao estado do Maranhão, dados estes vinculados ao portal SIVEP, outros estudos demonstraram que somente em 2009, este estado foi responsável por 5.685 mil casos/ano de um total de 5.800 mil casos/ano da Região Nordeste como um todo. Este estado apresenta características ambientais favoráveis à permanência de plasmódios, principalmente porque há bons criadouros naturais do anofelino vetor, além, há a exposição de grandes contingentes populacionais, contendo neste estado, áreas de alto, médio e baixo risco de infecção malárica. ${ }^{6,7}$

Em relação entre números de habitantes com os números notificados em cada estado observou-se que, no Piauí, apesar de ser o segundo estado com o maior número de casos, foi estado mais acometido, considerando esta relação, com aproximadamente 3 casos a cada 100 mil habitantes. Tal fato se deve, a distribuição de casos pelo número total de habitantes de cada estado, levando em consideração que, para o ano de 2019, estimou-se um número de 3.273.227 habitantes para o estado de Piauí, enquanto para o estado da Bahia, que apresenta o maior número de casos notificados para a região nordeste, a população estimada para o mesmo ano era de 14.873 .064 habitantes. $^{9}$

\section{CONSIDERAÇÓES FINAIS}

Os resultados deste estudo possibilitaram descrever o perfil epidemiológico da malária nos estados do Nordeste brasileiro, demostrando que apesar dos esforços do MS ainda encontra-se casos notificados sem o total controle da cadeia de transmissão da doença.

Para as áreas extra-amazônicas, como é o caso dos estados nordestinos, é essencial o mapeamento das áreas de risco, bem como a reavaliação das espécies 
DOI: 10.18606/2318-1419/amazonia.sci.health.v8n2p42-50 Revista Amazônia Science \& Health
GONÇALVES, CWB. RODRIGUES, RA. NETO, ABP. GOMES, DLF, SILVA' M. SORTE, GVB.

Análise dos Aspectos Epidemiológicos da Malária na região Nordeste do Brasil

vetores potencialmente incriminadas na transmissão, já que é contínua a expectativa da reintrodução do plasmódio e reemergência da malária em áreas hoje consideradas sob controle.

A limitação desta pesquisa foi o quantitativo de variáveis que apresentaram campos que não foram preenchidos corretamente pelas pessoas notificadas, o que dificulta uma análise mais efetiva dos dados. Observa-se nesse estudo dados importantes para o poder público delinear o aperfeiçoamento de estratégias e ações a fim de garantir o diagnóstico, tratamento e prevenção adequados para a população do estado. Em adição, afirma-se a necessidade de mais estudos a respeito do tema, no intuito de se obter uma análise mais refinada dos dados apresentados.

\section{REFERÉNCIAS}

1 Milner DA. Malaria Pathogenesis. Cold Spring Harb Perspect Med [internet]. 2018 jan [cited $2020 \quad$ abr 10$]$; 8(1). Available from: https://www.ncbi.nlm.nih.gov/pmc/articles/PMC5749143/pdf/cshperspectmed-MLRa025569.pdf

2 Braz RM, Andreozzi VL, Kale PL. Detecção precoce de epidemias de malária no Brasil: uma proposta de automação. Epidemiol Serv Saúde [internet]. 2006 jun [cited 2020 abr 10]; 15 (2): 21-33. Available from: http://scielo.iec.gov.br/pdf/ess/v15n2/v15n2a04.pdf

3 WHO - WORLD HEALTH ORGANIZATION. GLOBAL MALARIA PROGRAMME. World Malaria Report 2016. 2016.

4 Alburquerque BC, Martinez- Espinosa F, Suárez -Mutis MC. Malária. In: Dinâmica das doenças infecciosas e parasitárias. COURA JR. Rio de Janeiro: Guanabara Koogan, 2013; 885-910p.

5 Fontes CJF. Malária. In: SMI: Série de Medicina Interna- doenças infecciosas. PEDROSO ERP. Rio de Janeiro: Rubio; 2015. 399-424p

6 Brasil. Boletim Epidemiológico. Ministério da Saúde, Secretaria de Vigilância em Saúde [internet]. 2015 [cited 2020 abr 11]46 (43). Available from: https://www.saude.gov.br/images/pdf/2015/dezembro/16/2015-003---Mal--ria.pdf

7 Brasil. Situação epidemiológica da malária no Brasil, 2000 a 2011, Boletim Epidemiológico. Ministério da Saúde, Secretaria de Vigilância em Saúde [internet]. 2015 [cited 2020 abr 14]; 44(41). Available from: http://bvsms.saude.gov.br/bvs/periodicos/boletim_epidemiologico_numero_1_2013.pdf

8 Divep-Suvisa. Boletim Epidemiológico da Situação Epidemiológica da Malária no Estado da Bahia, 2018 - 2019. Secretária Estadual de Saúde - Bahia - Ano 07 № 01, 2019 
9 IBGE - INSTITUTO BRASILEIRO DE GEOGRAFIA E ESTATÍSTICA. População estimada:IBGE, Diretoria de Pesquisas, Coordenação de População e Indicadores Sociais, Estimativas da população residente com data de referência 10 de julho de 2019. 2019

10 Mendis K, Suna JB, Marchesini P, Carter R. The Negected burden of Plasmodium vivax malaria. American journal of tropical medicine and hygiene [internet]. 2001 jan [cited 2020 abr 14]; 64 (1): 97-106. Available from: http://www.ajtmh.org/docserver/fulltext/14761645/64/1_suppl/tmed_64-010097. pdf? expires $=1587343677 \& i d=i d \& a c c n a m e=$ guest $\&$ checksum $=1$ B8EDB78A9D4DAB BC5C487E7B6D87FE0 